J. Clin. Chem. Clin. Biochem.

Vol. 16, 1978, pp. 413-419

\title{
Untersuchung der Wechselwirkung von Carbamazepin und Promazin mit Rinderserumalbumin mit Hilfe einer neuen Dialysevorrichtung
}

\author{
Von $A$. Kinawi und C. Teller \\ Institut für Biochemie und Molekularbiologie (FB 23/WE 03) der Freien Universität Berlin
}

(Eingegangen am 9. September/9. Dezember 1977/28. März 1978)

Zusammenfassung: Der Aufbau und die Arbeitsweise einer neuen Dialysiervorrichtung werden beschrieben.

Die Brauchbarkeit dieser Vorrichtung, die die Bestimmung des freien Anteils eines Pharmakons in einer AlbuminPharmakon-Pufferlösung bereits nach 10-15 Minuten ermöglicht, wurde hier durch die Ermittlung des freien Anteils von Carbamazepin bzw. Promazin in Albumin-Pufferlösungen demonstriert. Die hierbei erzielten Ergebnisse zeigten eine gute Ubereinstimmung mit denen der Kontrollmethoden (Gleichgewichtsdialyse und Gelfiltration). Weiterhin wurde die Carbamazepin-Albumin-Bindung durch Ermittlung der Gesamtbindungskonstante $\left(\mathrm{K}_{1}\right)$, der scheinbaren (apparenten) Bindungskonstante $\left(\mathbf{k}^{+}\right)$, der Anzahl der Bindungsstellen/Albuminmolekül ( $\left.\mathbf{n}\right)$, der freien Reaktionsenergie $\Delta \mathrm{F}^{0}$ sowie des freien Anteils charakterisiert.

Investigation of the interaction of Carbamazepine or Promazine with bovine serum albumin, using a new dialysis device

Summary: The construction and operation of a new dialysis device are described.

The unbound fraction of a drug in a buffered solution of albumin can be determined after 10-15 minutes. The usefullness of this device was demonstrated by the determination of the unbound fraction of Carbamazepine or Promazine in buffered solutions of bovine serum albumin. The results agreed with those obtained by control methods (equilibrium dialysis and gel filtration). Furthermore, the binding of Carbamazepine to bovine serum albumin was characterised by determining the overall binding constant $\left(\mathrm{K}_{1}\right)$, the apparent binding constant $\left(\mathrm{k}^{\dagger}\right)$, the number of binding sites per molecule of albumin (n), the free reaction energy $\Delta \mathrm{F}^{\circ}$, and the unbound fraction of the drug.

\section{Einleitung}

Im Rahmen von Unterșuchungen über die Inkompatibilität und Interaktion von Antikoagulantien und Psychopharmaka ergab sich die Frage nach der Bindung bzw. der Änderung der Bindungsverhältnisse dieser Pharmaka an Serumproteine. Bindungsștudien könnten eventuell, falls ein Pharmakon bzw. einer seiner Metabolite die Bindungsverhältnisse eines anderen Pharmakons ändern, zur Klärung des Mechanismus der von uns in Tierversuchen untersuchten Interaktion (1-5) beitragen. Diẹ Literaturfülle der letżen żehn Jahre auf dem Gebiet der Bestimmung des freien Anteils eines Pharmakons in biologischem Material bietet hinsichtlich der angewandten Methoden ein einheitliches Bild:

Benutzt wurden
a) Gelfiltration
b) Gleichgewichtsdialyse
c) Ultrafiltration/-zentrifugation $(12,22-30)$

Ohne hier näher auf die Vor- und Nachteile der Gelund Ultrafiltration bzw. Ultrazentrifugation einzugehen (12), soll mit der vorliegenden Arbeit gezeigt werden, wie der wichtigste Nachteil der Gleichgewichtsdialyse, nämlich die UUberlänge der Versuchsdauer (2-17 h) auf ein Minimum von 10-15 Minuten reduziert werden kann. Dazu wurde die Bindung von Carbamazepin und Promazin an Rinderserumalbumin mit Hilfe einer neuen Dialysevorrichtung untersucht.

\section{Material and Methoden}

Bindungstudien $\mathrm{m}$ it $\mathrm{H}$ ilfe der $\mathrm{Z}$ irkulationsvorrichtung

\section{Material}

1. Phosphatpuffer nach S申rensen ( $\mathrm{pH} 7,4$ )

2. $150 \mathrm{ml}$ Rinderserumalbuminlösung $(40 \mathrm{~g} / \mathrm{l})$ in Phosphatpuffer. Da der Reinheitsgrad nach Angaben des Herstellers (Merck) $90 \%$ beträgt, enthielten die hier verwendeten Lösungen $1,11 \mathrm{~g} / 25 \mathrm{ml}$ Rinderalbumin. 
3. Carbamazepin-Pufferlösung der Konzentration: $0,5,1,2,3$, 5 und $10 \mathrm{mg} / \mathrm{l}$, (für die Erstellung der Bezugskurve).

4. Lösungen von $5,10,15,20,25$ und $30 \mathrm{mg} / 1$ Carbamazepin in Albumin-Pufferlösung (je $25 \mathrm{ml}$ ). Die erforderlichen Carbamazepinmengen wurden durch Eindampfen entsprechender Volumina einer methanolischen Carbamazepinlösung $(1 \mathrm{~g} / \mathrm{l})$ erhalten.

5. Etwa $1 \mathrm{~m}$ Tygon-Schlauch (1/16" i. D., 1/8" ä. D.)

6. $20 \mathrm{~cm}$ Visking-Dialysierschlauch, $\mathrm{s}$. unten, $(1 \mathrm{~h}$ zuvor in Puffer liegen lassen, dann an den Knickstellen in zwei Membranen geschnitten).

Geräte

1. Die hier beschriebene Dialysiervorrichtung mit Ventilen und Luftabscheidern.

2. Eine Schlauchpumpe (Desaga-Vielfach-Schlauchpumpe 131900).

3. Spektralphometer mit einer Mikroküvette (8 $\mu \mathrm{l}$-Volumeninhalt, Varian, Techtron-Spektralphotometer Modell Nr. 635). Es wurde bei $280 \mathrm{~nm}$ gemessen, Empfindlichkeit $A=0-0,1$.

4. Ein Schreiber (Varian $25 \mathrm{~A}$, bei $50 \mathrm{mV}, 20 \mathrm{~cm} / \mathrm{h}$ ). Schaltschema s. Abbildung 1.

An dieser Stelle muß jedoch darauf hingewiesen werden, daß auf Abbildung 1 der Anschaulichkeit halber 2 bzw. 3 Pumpen verwendet wurden. In der Praxis kommt man jedoch mit einer Pumpe aus.

Prinzip (Abb. 1)

Die enorme Zeitersparnis beruht auf folgender Änderung bei der Durchführung der Gleichgewichtsdialyse:

1. Die beiden Flüssigkeitsmengen, zwischen denen sich das Gleichgewicht einstellen soll, werden nicht, wie üblich, geschüttelt oder gerührt, sondern sie durchströmen zirkulierend (im Gegenstrom) zwei voneinander durch eine Dialysemembran getrennte Kammern.

2. Die Zunahme der Konzentration des zu bestimmenden Stoffes in dem Stromzyklus der einen Kammer wird mit Hilfe eines in den Zyklus integrierten Detektors fortlaufend registriert.

3. Der am Detektor (UV-Mikroküvette) angeschlossene Schreiber registriert die zeitliche Konzentrationszunahme.
4. Es wird nicht bis zum Ende der Einstellung des Gleichgewichts gewartet, sondern die „Zirkulationsgleichgewichtsdialyse" wird nach 10-15 Minuten unterbrochen (siehe Diskussion).

5. Da mit der gleichen Arbeitsweise die Bezugskurve für den zu bestimmenden Stoff erstellt wird (s. unten), entfällt die Bestimmung des Stoffanteils, welcher an die Membran gebunden wird. Hierdurch entfält der zweite Nachteil der Gleichgewichtsdialyse.

\section{Beschreibung dẹr Vorrichtung (Abb. 2)}

Die Vorrichtung besteht aus zwei Plexiglasplatten (A) und (B) $100 \times 30 \times 10 \mathrm{~mm}$ (Abb. $2 \mathrm{a})$, die zwei Teflonplatten $(100 \times 30$ $\times 0,5 \mathrm{~mm}$ ) umschließen.

Die Teflonplatten (D) weisen einen Schlitz von $70 \mathrm{~mm}$ Länge und $4 \mathrm{~mm}$ Breite auf (Abb. 2b). Die beiden Teflonplatten schließen ihrerseits eine Dialysemembran (C) (Visking 8/32) ein (gestrichelte Linie in Abb. 2a). Somit entstehen zwei durch die Dialysiermembran getrennte Kammern. Wie Abbildung $2 b$ zeigt, wird jede dieșer Kàmmern mit Hilfe von zwei durchbohrten Schrauben (E) (aus Delrin) nach außen verbunden. Die beiden Platten (A) und (B) werden mit Hilfe von acht Schrauben miteinander befestigt.

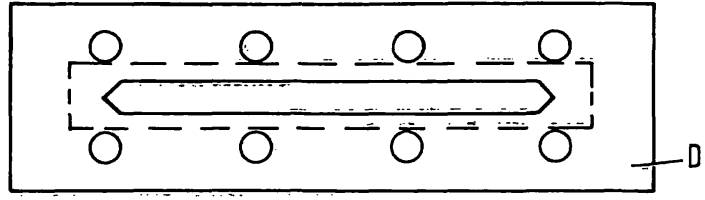

Abb. 2a. Eine der beiden Teflonplatten mit Bohrungen und Schlitz. Gestrichelte Linie zeigt die Membran an.

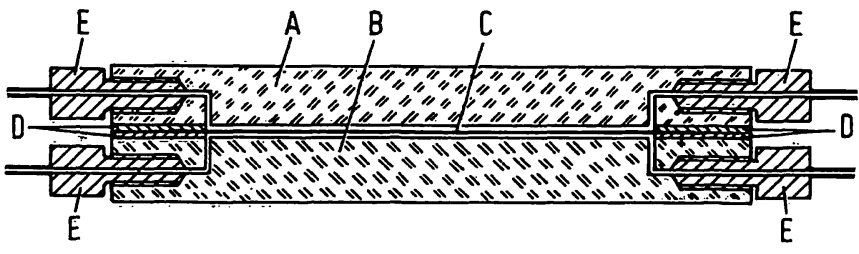

Abb. 2b. Schnitt längs der Zirkulationsgleichgewichtsdialysevorrichtung, A, B und $E$ siehe Text.

C. Dialysemembran.

D. Teflonplatten.

\section{Bemerkung zur Handhabung}

Der Inhalt einer der beiden Kammern (Pharmakon-Puffer- bzw. Pharmakon-Puffer-Albuminlösung) zirkuliert mit Hilfe einer Schlauchpumpe $(P)$, die über einen Luftabscheider $\left(\mathrm{L}_{1}\right)$ und ein Ventil $\left(V_{1}\right)$ am Ein- und Ausgang der Kammer angeschlossen ist (s. schematische Darstellung Abb. 1 sowie Abb. 3).

Der Inhalt der zweiten Kammer (Pufferlösung) zirkuliert durch das Ventil $\left(V_{2}\right)$, zwei Luftabscheider $\left(\mathrm{L}_{2}, \mathrm{~L}_{3}\right)$ und den Detektor (D). Die Luftabscheider werden am Eingang der Kammer bzw. des Detektors angebracht und sind notwendig, um die Kammern und vor allen Dingen den Detektor, welcher sehr empfindlich auf Luftbläschen reagiert, luftfrei zu halten. Außerdem dienen sie zum Volumenausgleich beider Zyklen. Der Luftabscheider $\left(L_{2}\right)$ enthält ein Filter aus Polyethylen. Dieses verhindert das Einsaugen von Verunreinigungen und bewirkt einen geringen Überdruck im Detektor, wodurch hier die Luftblasenbildung gehemmt wird. Dieses Filter muß bei leichter Verstopfung, erkenntlich an vermehrter Blasenbildung in der Verbindung zwischen Kammerausgang und Pumpeneingang, jeweils ausgetauscht werden.

Vor jëder Messung muß strengstens darauf geachtet werden daß der Schreiber die Nullinie einhält (kein Driften), sonst sind die Dialysiermembran und die Verbindungsschläuche auszutauschen (siehe weițer unten).
Abb. 1. Schaltschema: $P=$ Pumpe, $V_{1}$ und $V_{2}=$ Ventile, $L_{1-3}=$ Luftabscheider, $D=$ Detektor, $S=$ Schreiber.

Teile A und B s. Abb. 2 a. 


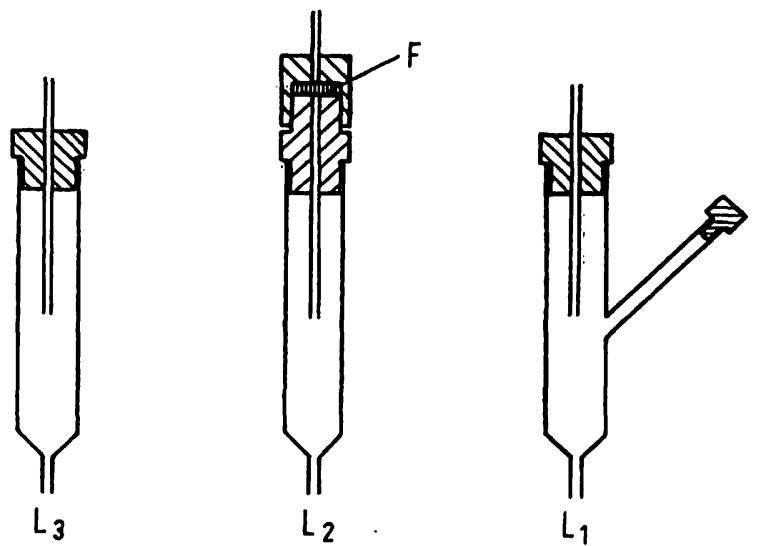

Abb. 3. Schnitt längs der Luftabscheider. $\mathbf{F}=$ Filter.

Bestimmung des freien Anteils von Carbamazepin (Tegretal, 5-Carbamoyl-5-H-dibenz-[b, f]-azepin) in $R$ inderserumalbumin-Carbamazepin-Pufferlösungen

Der freie Anteil von Carbamazepin in RinderserumalbuminCarbamazepin-Pufferlösungen (s. unten) wurde mit Hilfe des oben beschriebenen Verfahrens bestimmt. Um die Genauigkeit dieser Methode überprüfen zu können, wurde der freie Anteil weiterhin mit Hilfe der Gelfiltration und der Gleichgewichtsdialyse ermittelt.

\section{Durchfihrung der Bestimmung}

Die Vorrichtung wurde zuerst über die geöffneten Ventile mit etwa 50-70 ml der Pufferlösung ausgespïlt. Durch Umschalten der Ventile wurden die Stromkreise geschlossen und der Inhalt beider Zyklen solange in Bewegung gehalten, bis der Schreiber keine Störung zeigt.

Bei laufender Pumpe $(375 \mathrm{ml} / \mathrm{h}$ ) wurde nun der Inhalt der oberen Kammer über $\mathrm{V}_{1}$ entleert, der Kreis wieder geschlossen, $1,8 \mathrm{ml}$ der Carbamazepin-Pufferlösung $(0,5 \mathrm{mg} / 1)$ durch $\mathrm{L}_{1}$ (s. Abb. 1) in den Kreislauf eingespritzt.

Nach etwa 10-15 Minuten wurde der Inhalt beider Zyklen über $V_{1}$ und $V_{2}$ mit Puffer verdrängt. Um die Carbamazepinspuren zu entfernen, wurde soviel Puffer $(50-70 \mathrm{ml})$ durch das System gesaugt, bis der Schreiber auf die Nullinie zurückging und diese Stellung einhielt. Anschließend wurde der oben beschriebene Vorgang mit derselben Carbamazepin-Lösung $(0,5 \mathrm{mg} / \mathrm{l})$ żweimal wiederholt.

Mit den anderen Carbamazepin-Pufferlösungen, die wegen der schlechten Löslichkeit von Carbamazepin in Puffer zuvor auf ihren Gehalt an gelöster Substanz photometrisch kontrolliert werden müssen, wurde entsprechend verfahren. Das Volumen von $1,8 \mathrm{ml}$ entspricht dem Volumeninhalt des unteren Zyklus.

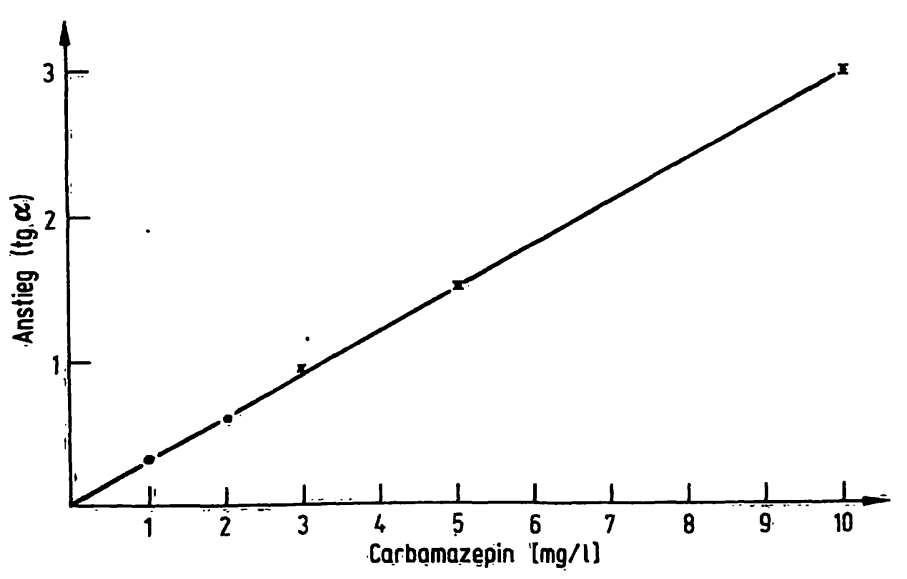

Abb. 4. Bezugskurve fự Garbamazepin bei $280 \mathrm{~nm}$.
Zur graphischen Darstellung der Bezugskurve (Abb. 4) wurde der Anstieg gegen die entsprechenden Carbamazepinkonzentrationen aufgetragen. Zur Bestimmung des nicht gebundenen Anteils von Carbamazepin in Rinderserumalbumin-CarbamazepinPufferlösungen wurden diese Lösungen analog der CarbamazepinPufferlösungen bei der Erstellung der Bezugsgeraden behandelt (je Probe drei Bestimmungen). Aus dem ermittelten Anstieg wurde der freie Anteil an Carbamazepin anhand der Bezugskurve bestimmt.

Bestimmung des nicht gebundenen Anteils von Promazin (10-3'-dimethylaminopropyl)-phenothiazin)

in $R$ inderserumalbumin-Promazin-Pufferlö sungen

Promazin gehört zu den ersten Phenothiazinderivaten, die von Krieglstein \& Kuschinsky hinsichtlich der Bestimmung des freien Anteils in Eiweißlösungen mit Hilfe der Gelfiltration untersucht worden sind (31). Die erzielten Ergebnisse wurden, um die Brauchbarkeit der Gelfiltration in diesem Zusammenhang zu demonstrieren, mit den entsprechenden Ergebnissen der Gleichgewichtsdialyse und Ultrazentrifugation verglichen.

Die gute UUbereinstimmung der Ergebnisse mit den drei Bestimmungsmethoden (Gelfiltration, Gleichgewichtsdialyse und Ultrazentrifugation) hat uns veranlaßt, die Bestimmung des freien Anteils von Promaz in in Albumin-Pufferlösungen mit Hilfe der hier beschriebenen Methode durchzuführen. Hierzu wurde analog der Bestimmung des freien Anteils von Carbamazepin in Albumin-Pufferlösungen verfahren (siehe oben).

Sowohl die Carbamazepin- als auch Promazin-Rinderserumalbumin-Pufferlösungen wurden auf ihren Gehalt an Pharmakon kontrolliert. Es wurde hierbei analog der Bestimmung des Gehaltes der Einzelfraktionen an Carbamazepin bei der Gelfiltration verfahren (siehe oben).

Bindungsstudien $\mathrm{m}$ it $\mathrm{H}$ ilfe der Gelfiltration

Säule $\quad 15 \times 1,2 \mathrm{~cm}$

Stationäre Phase Sephadex G 25 fein (Deutsche Pharmacia GmbH Frankfurt/Main)

Mobile Phase Phosphatpuffer pH 7,4 (s. unten), $15 \mathrm{ml} / \mathrm{h}$

Probenvolumen $13 \mathrm{ml}$

Der Gehalt der Fraktionen (je $3 \mathrm{ml}$ ) an Carbamazepin wurde durch Extraktion mit Methylenchlorid ( $3 \times$ je 3 ml-Fraktion), Eindampfen der vereinigten Extrakte, Lösen der Rückstände in Methanol, Messen der Absorption der methanolischen Lösungen bei 280 und $230 \mathrm{~nm}$ und an Hand von Standardgeraden für Carbamazepin ermittelt.

Bindungsstudien mit Hilfe der Gleichgewichtsdialyse Hierzu wurde das von Niedner et al angegebene Dialysiersystem verwendet (21). (Visking Dialysierschläuche $8 / 32,7 \mathrm{~mm}$ Durchmesser, $1 \mathrm{ml}$ Probe). Die Dialysierzeit wurde aus arbeitstechnischen Gründen auf 17 Stunden ausgedehnt, obwohl Vorversuche gezeigt haben, daß bereits nach 5-7 Stunden die Gleichgewichtslage zu $99,5 \%$ erreicht wird.

\section{Ergebnisse}

Die erzielten Ergebnisse lassen sich zu folgenden Punkten zusammenfassen:

1. Der freie Anteil an Carbamazepin (bei Raumtemperatur von $22^{\circ} \mathrm{C}$ ) ist der Tabelle $1 \mathrm{zu}$ entnehmen.

2. Der freie Anteil an Promazin (Raumtemperatur $22^{\circ} \mathrm{C}$ ) ist der Tabelle $2 \mathrm{zu}$ entnehmen.

3. Die Diffusionsgeschwindigkeit von Carbamazepin durch die Dialysemembran ist ausreichend. So nahm eine Ausgangskonzentration von $10 \mathrm{mg} / \mathrm{l}$ Carbamazepin in Puffer bereits nach 10 Minuten Zirkulationsdauer um $11 \% \mathrm{ab}$. 
Tab. 1. Die ermittelten freien Anteile von Carbamazepin in Rinderserumalbumin-Lösung ( $40 \mathrm{~g} / \mathrm{l}$ ) in Abhängigkeit von der Carbamazepingesamtkonzentration (A) und der Bestimmungsmethoden.

\begin{tabular}{llll}
\hline A & Stoffmengenverhältnis & \multicolumn{2}{c}{ Carbamazepin frei } \\
\cline { 3 - 4 } [mg/l] & $\begin{array}{l}\text { Gleich- } \\
\text { gewichts- } \\
\text { dialyse }\end{array}$ & Gelfiltration & $\begin{array}{l}\text { hiepin be- } \\
\text { schriebene } \\
\text { Methode }\end{array}$ \\
\hline 5 & 0,245 & 0,239 & 0,238 \\
10 & 0,250 & 0,240 & 0,240 \\
15 & 0,248 & 0,241 & 0,240 \\
20 & 0,249 & 0,241 & 0,240 \\
25 & 0,252 & 0,242 & 0,241 \\
30 & 0,253 & 0,242 & 0,241 \\
\hline
\end{tabular}

Tab. 2. Die ermittelten freien Anteile von Promazin in Rinderserumalbumin-Pufferlösung ( $40 \mathrm{~g} / \mathrm{l})$ in Abhängig̈keit von der Bestimmungsmethode.

\begin{tabular}{ll}
\hline Bestimmungsmethode & Promazinfrei \\
\cline { 2 - 2 } & Promazin $_{\text {gesamt }}$ \\
\hline${\text { Zirkulationsmethode (bei } 22^{\circ} \mathrm{C} \text { ) }}_{\left.\text {Gelfiltration }^{1} \text { ) (bei } 22^{\circ} \mathrm{C}\right)}$ & $0,330 \pm 0,003$ \\
Gleichgewichtsdialy se $^{1}$ ) (bei $\left.22^{\circ} \mathrm{C}\right)$ & $0,333 \pm 0,003$ \\
Ultrazentrifugation $^{1}$ ) (bei $\left.4{ }^{\circ} \mathrm{C}\right)$ & $0,337 \pm 0,012$ \\
\hline
\end{tabular}

1) Diese Angaben dienen zum Vergleich mit den durch die Zirkulationsmethode erhaltenen Ergebnissen und sind der Literatur entnommen (31).

4. Die Reproduzierbarkeit der Meßergebnisse ist Tabelle $1 \mathrm{zu}$ entnehmen. Die angegebenen Werte für den freien Anteil stellen unter Verwendung der Zirkulationsvorrichtung gefundene Mittelwerte von je drei Bestimmungen dar. Die Streuung (Abweichung vom Mittelwert) lag hier bei $\pm 1,5 \%$ (bei $5 \mathrm{mg} / 1$ Carbamazepin). Dieser Wert nahm jedoch bei zunehmender Carbamazepinkonzentration ab $( \pm 0,5 \%$ bei $30 \mathrm{mg} / \mathrm{l}$ ).

5. Silikonschläuche geben trotz vorheriger Reinigung mit heißem Wasser ständig unbekannte Verunreinigungen an den zirkulierenden Puffer ab (fortlaufende Entfernung des Schreibers von der Nullinie). Bei den hier verwendeten Tygon-Schläuchen genügt eine Reinigung mit heißem Wasser $\left(70^{\circ} \mathrm{C}\right)$, um diese Störung zu vermeiden.

6. Die Verdopplung der Schlitzbreite in den beiden Teflonstreifen fuihrte nicht zur Verdopplung des Anstieges. Dies ist auf die Entstehung von nichtlaminaren Strömungen zurückzuführen. Eine Schlitżbreite von 4-5 $\mathrm{mm}$ erwies sich als optimal.

\section{Charakterisierung}

der Carbamazepin-Rinderserumalbumin-Bindung

Hierzu wurden die Gesamtbindungskonstante $K_{1}$, die Anzahl der Bindungsstellen n, die freie Reaktionsenergie $\Delta \mathrm{F}^{0}$, die. Steigungskonstante $\mathrm{m}$ und die scheinbare (apparente) Bindungskonstante $\mathbf{k}^{+}$ermittelt.

\section{Bestimmung von $\mathrm{K}_{1}$}

Die Versuchsergebnisse, dargestellt nach Scatchard (32), ergeben eine gekrümmte Linie (Abb. 5). Dies ist ein Hinweis auf das Vorliegen von mindestens zwei verschiedenen Arten von Bindungsorten, die sich in ihrer Affinität zu Carbamazepin unterscheiden.

Der Schnittpunkt der verlängerten Linie mit der Ordinate (Abb. 5) gibt die Gesamtbindungskonstante $K_{1}$ an. Hieraus läßt sich für $K_{1}$ ein Wert von: $5,53 \times 10^{3} 1 / \mathrm{mol}$ ermitteln. Werden die Quotienten [gebundener Anteil]/ [freier Anteil], $\left(C_{g} / C_{f}\right)$ gegen $C_{f}$ aufgetragen, erhält man eine gekrümmte Linie, deren Verlängerung die Ordinate $\left(\mathrm{C}_{\mathbf{g}} / \mathrm{C}_{\mathbf{f}}\right)$ bei einem der Gesamtbindungskonstante proportionalen Wert schneidet. Durch Dividieren dieses Wertes durch die molare Konzentration der verwendeten Albuminlösung erhält man die Gesamtbindungskonstante (33). In dem hier behandelten Fall ergab sich eine gute Übereinstimmung. Es ließ sich ein $\mathrm{K}_{1}$-Wert von $5,51 \times 10^{3} \mathrm{l} / \mathrm{mol}$ errechnen.

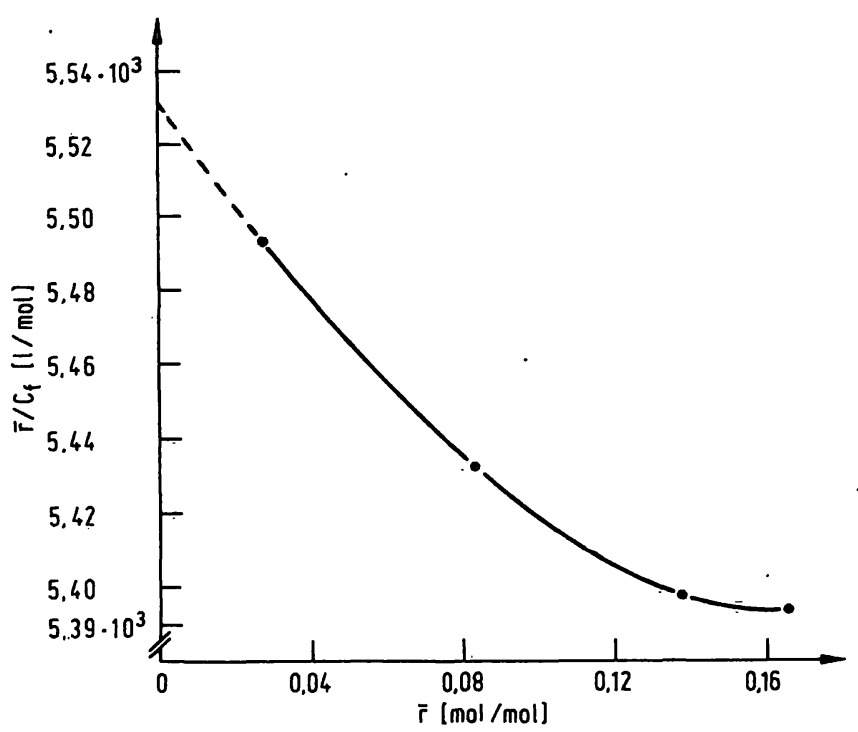

Abb. 5. Abhängigkeit der Quotienten $\overline{\mathrm{r}} / \mathrm{C}_{\mathrm{f}}$ von $\overline{\mathrm{r}},(\overline{\mathrm{r}}=$ spezifisches Bindungsvermögen in $\mathrm{mol} / \mathrm{mol}, \mathrm{C}_{\mathrm{f}}=$ Konzentration des freien Anteils an Carbamazepin in mol/l) (zur Bestimmung der Gesamtbindungskonstanten $\hat{K}_{1}$ ).

\section{Bestimmung von $n$}

Der Schnittpunkt der Kurve mit der Abszisse in Abbildung 5 sollte die Anzahl der Bindungsstellen n/Albuminmolekül angeben (32). Da wir aber, um möglichst im pharmakologisch interessierenden Konzentrationsbereich zu bleiben, nicht die Sättigung aller möglichen Bindungsstellen angestrebt haben, verlief hier die Kurve fast parällèl żur Abszisse. Die Bestimmung von n/Albuminmolekül erfolgt in diesem Fall durch Auftragung der reziproken Werte des spezifischen Bindungsvermögens $(1 / \bar{r})$ gegen den reziproken Wert der Konzentration des freien Anteils $\left(1 / C_{f}\right)$. Der Schnittpunkt der Geraden mit der Ordinaten $(1 / \bar{r})$ gibt den reziproken Wert für $n$ an. (Abb. 6). Es wurden für Carbamazepin fünf Bin- 


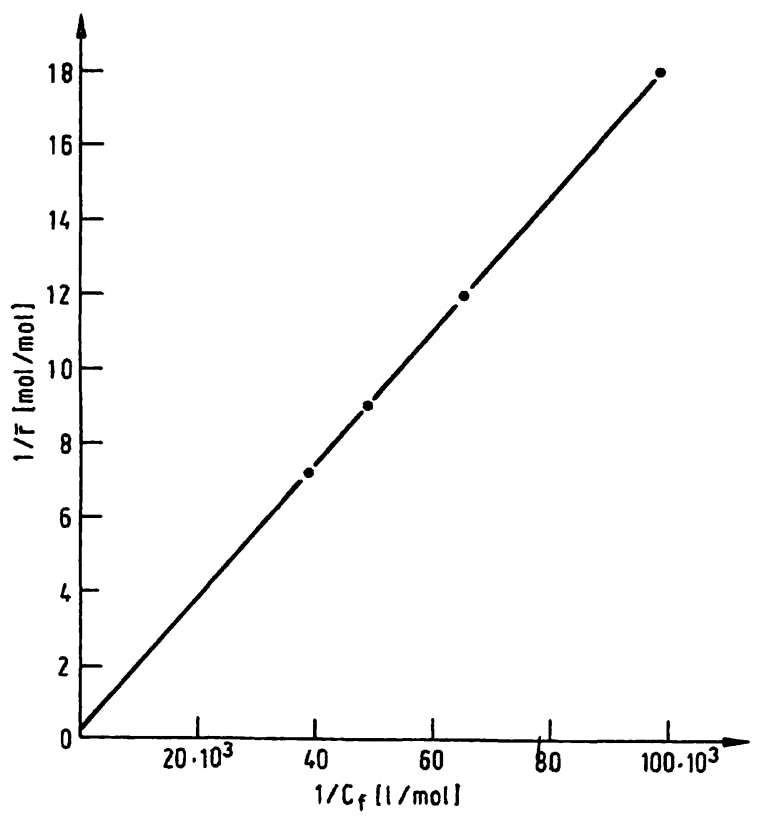

Abb. 6. Abhängigkeit des reziproken Wertes des spezifischen Bindungsvermögens $\bar{r}$ vom rezjproken Wert der freien Carbamazepinkonzentration $\mathrm{C}_{\mathbf{f}}$ (zur Bestimmung der Anzahl der Bindungsstellen (n) pro Albuminmolekül).

dungsstellen am Rinderserumalbumin-Molekül bestimmt.

\section{Bestimmung von $m$ und $k^{+}$}

Beide Größen lassen sich durch die Auftragung von $\lg C_{g}$ gegen $\lg C_{f}$ bestimmen (34). Ergibt die Auftragung eine Gerade (Abb. 7), so ist die Gleichung:

$$
\lg C_{g}=\operatorname{lg~k}+m \lg C_{f} \text { bzw. } k^{+}=C_{g} / C_{f}^{m}
$$
erfüllt.

Der Anstieg der erhaltenen Geraden (Abb. 7) entspricht $\mathrm{m}=0,976$; hieraus ließ sich für $\mathrm{k}^{+}$ein Wert von $1,36 \times 10^{-5} \mathrm{~mol}^{1-\mathrm{m}}$ errechnen.

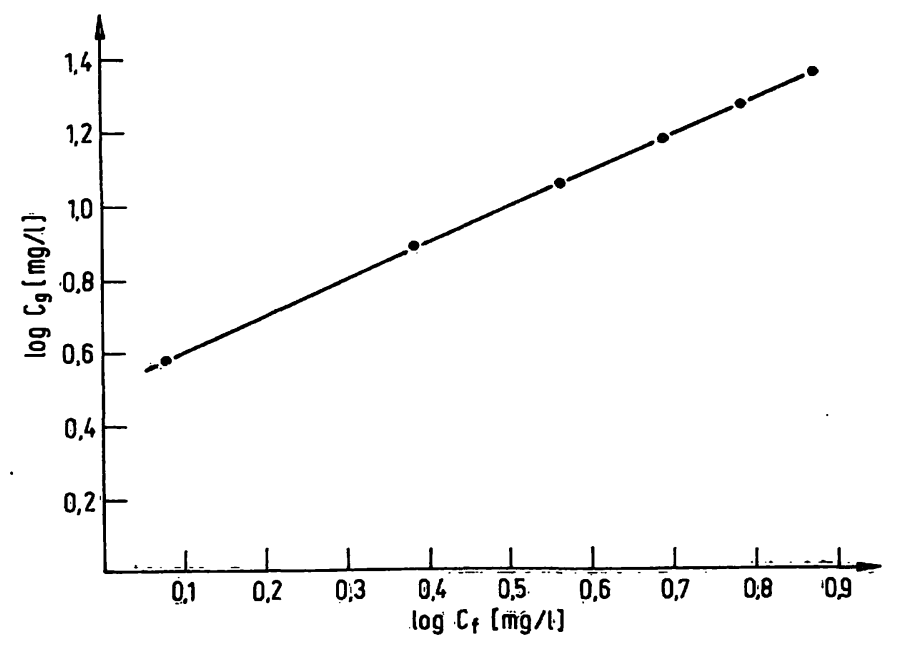

Abb. 7. Auftragung von $\log \ddot{C}_{g}$ gegen $\log \hat{C}_{f}$ zur Ërmittlung der Steigungskonstanten $\mathrm{m}$ und der scheinbaren (apparenten) Bindungskonstanten $\mathbf{k}^{\mathbf{+}}$.

\section{Bestimmung von $\Delta F^{0}$}

Dies erfolgt nach der Gleichung:

$$
\Delta \mathrm{F}^{0}=-\mathrm{RT} \ln \mathrm{K}_{1}
$$

Für

$$
\mathrm{K}_{1}=5,53 \cdot 10^{3} 1 / \mathrm{mol}
$$

ist

$$
\Delta \mathrm{F}^{0}=-5053 \mathrm{cal} / \mathrm{mol} \quad(-21,16 \mathrm{~kJ} / \mathrm{mol})
$$

Für

$$
\mathrm{K}_{1}=5,51 \cdot 10^{3} \mathrm{l} / \mathrm{mol}
$$

ist

$$
\Delta \mathrm{F}^{0}=-5051 \mathrm{cal} / \mathrm{mol} \quad(-21,14 \mathrm{~kJ} / \mathrm{mol})
$$

\section{Diskussion}

Die Brauchbarkeit des hier verwendeten Dialysesystems wurde anhand der Bestimmung des freien Anteils von Carbamazepin bzw. Promazin in Albumin-Pufferlösungen demonstriert. Als Nachteil dieser Methode kann ihre Anfälligkeit gegen Luftblasen und Verunreinigung der Verbindungsschläuche und der Membran gesehen werden, die sich jedoch augenfällig bemerkbar machen.

Pharmaka als niedermolekulare Verbindungen zeigen zwar ein differenziertes Diffusionsvermögen durch eine gegebene Membran; dennoch ist dies nicht der primäre Grund für die unterschiedliche Länge der Dialysedauer von Eiweiß-Pharmakalösungen. Die Ursache hierfür ist vor allem in der Natur der Eiweiß-PharmakonBindung zu suchen. Je stärker diese Bindung ist, um so langsamer erfolgt der Entzug des Pharmakons aus der Sphäre des bindenden Proteins und um so länger dauert die Gleichgewichtsdialyse.

Nach eigener Beobachtung (noch nicht publizierte Ergebnisse) ist die Zeitdauer bis zur Wiederherstellung des Gleichgewichtes Albumin + Pharmakon $\rightleftharpoons$ Pharmakon Albuminkomplex (nach raschem Entfernen des freien Anteils) von der Struktur der Pharmaka abhängig. Im Durchschnitt liegt diese Zeitdauer bei 10-60 Minuten. Somit verläuft die Gleichgewichtsdialyse in der ersten Phase weitgehend auf Kosten des freien Anteils, worauf die hier beschriebene Methode beruht, zumal im Gegenstrom dialysiert wird.

Wird z. B. unter Anwendung des hier beschriebenen Dialysiersystems ein gegebenes Volumen einer Pharmakon-Pufferlösung $(10 \mathrm{mg} / \mathrm{l})$ gegen das gleiche Volumen desselben Puffers bis zur Einstellung des Gleichgewichtes dialysiert, so registriert der Schreiber eine Sättigungskurve, deren Anfang eine ansteigende Gerade darstellt. Der Anstieg dieser Geraden ist abhängig von:

1. der Ausgangskonzentration des Pharmakons im Puffer

2. dem spezifischen Diffusionsvermögen des Pharmakons durch die gegebene Membran 
3. dem Absorptionsvermögen dieser Membran für das Pharmakon

4. der bei der Messung verwendeten Wellenlänge

5. dem Papiertransport/min.

Wird nun unter Konstanthal tung der Versuchsbedingungen statt der Pharmakonpufferlösung $(10 \mathrm{mg} / \mathrm{l})$ eine Pharmakon-Albumin-Pufferlösung dialysiert, wobei die Konzentration des nicht gebundenen Pharmakons ebenfalls $10 \mathrm{mg} / 1$ beträgt, (als Beispiel betrachten wir eine Lösung von $100 \mathrm{mg} / \mathrm{l}$ Pharmakon in Albuminpufferlösung), so würde der Schreiber die gleiche Sättigungskurve wie bei der Dialyse der Pharmakon-Pufferlösung ( $10 \mathrm{mg} / \mathrm{l})$ registrieren, wenn die Eiweiß-Pharmakon-Bindung während der Dialyse nicht teilweise zerfallen würde. Da aber trotz des Zerfalls der PharmakonProtein-Bindung und der damit verbundenen ständigen Zufuhr an nicht gebundenem Pharmakon die Ausgangskonzentration des freien Pharmakons ständig abnimmt (unter Vernachlässigung der Membranadsorption sinkt sie bei der Einstellung des Gleichgewichtes auf $9,09 \mathrm{mg} / \mathrm{l}$ ), registriert der Schreiber hier ebenfalls den gleichen Anstieg wie bei der Dialyse der PharmakonPufferlösung gleicher Ausgangskonzentration. Beide Sättigungskurven zeigen also den gleichen Anstieg worauf die Bestimmungsmethode beruht - und unterscheiden sich lediglich in der Länge der ansteigenden Geraden. Dieser Unterschied ist auf die unterschiedliche Gesamtzunahme des diffundierenden Pharmakons im Puffer zurückzuführen.

Die gute Ǔbereinstimmung der mit diesem Dialysiersystem erzielten Ergebnisse mit denen der Kontrollmethoden (Gelfil tration, Gleichgewichtsdialyse) läßt annehmen, daß die ihrerseits konzentrationsabhängige Adsorption des Pharmakons an der Membran die Bestimmungsmethode nicht stört.

Die Bestimmung der Bindungsstellen für ein Pharmakon am Eiweißmolekül unter Berücksichtigung pharmakologisch interessierender Konzentrationsverhältnisse ist kaum zu realisieren, da eine zuverlässige Bestimmung der Anzahl dieser Stellen eine Erschöpfung der Bindungskapazität des Eiweißes für das Pharmakon vor-

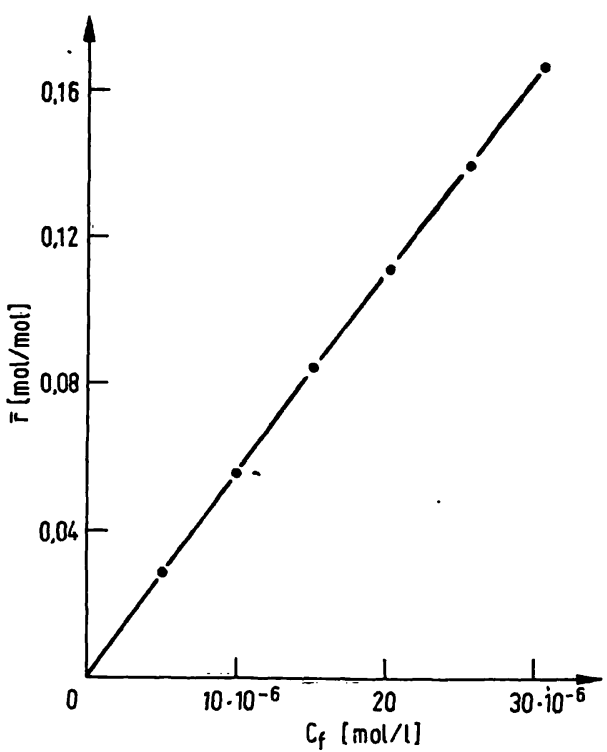

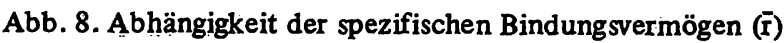
von der freien Carbamazepinkonzentration $\left(C_{f}\right)$.

aussetzt. In dem hier behandelten Fall von Carbamazepin ist dieser Zustand selbst bei einer Carbamazepingesamtkonzentration von $30 \mathrm{mg} / 1$ Eiweißlösung bei weitem noch nicht erreicht (Abb. 8), obwohl diese Konzentration dem 3-5-Fachen der unter therapeutischen Bedingungen üblichen Konzentration entspricht. Abgesehen davon, daß die Erhöhung der gebundenen Pharmakonmenge/Eiweißmolekül eine Konfigurationsänderung des Eiweißmoleküls verursacht (35), wodurch eine Änderung der Bindungskapazität des Eiweißes bedingt ist, bestimmt die geringe Löslichkeit der Pharmaka im Puffer den erreichbaren Sättigungsgrad des Eiweißes mit dem Pharmakon. Aus diesen Gründen ist die hier ermittelte Anzahl an Bindungsstellen für Carbamazepin/Molekül-Albumin lediglich als eine unter den gegebenen Versuchsbedingungen ermittelte Größe anzusehen.

Die hier gezeigte Dialyse-Vorrichtung eignet sich weiterhin für Gleichgewichtsstudien im offenen System (Fließgleichgewicht). Hierfür wird (s. Abb. 1, gestrichelter Teil) eine zweite Dialyse-Vorrichtung angeschlossen.

\section{Literatur}

1. Kinawi, A. \& Baumgartl, I. (1977), J. Clin. Chem. Clin. Biochem. 15, 345-348.

2. Kinawi, A., Döring, B. \& Witte, I. (1977), Arzneim.-Forsch. 27, 747-750.

3. Kinawi, A., Onken, A., Rozyczka, B. \& König, W. (1977), Arzneim.-Forsch. 27, 363-367.

4. Kinawi, A. \& Baumgartl, I. (1976), Arzneim.-Forsch. 26, 2019-2023.

5. Kinawi, A. (1977), Arzneim.-Forsch. 27, 360-363.

6. Hüthwohl, B. \& Jähnchen, E. (1972), Naunyn-Schmiedebergs Arch. Pharmakol. 273, 204-212.

i. Jähnchen, E. \& Krieglstein, J. (1971), Naunyn-Schmiedebergs Arch. Pharmakol. 268, 300-309.

8. Glasser, H. \& Krieglstein, J. (1970), Naunyn-Schmiedebergs Arch. Pharmakol. 265, 321-334.

9. Franz, J. W., Jähnchen, E. \& Krieglstein, J. (1969), NaunynSchmiedebergs Arch. Pharmakol. 264, 462-475.

10. Krieglstein, J. \& Kuschinsky, G. (1969), Naunyn-Schmiedebergs Arch. Pharmakol. 262, 1-16.

11. Jähnchen, E., Krieglstein, J. \& Kuschinsky, G. (1969), Naunyn-Schmiedebergs Arch. Pharmakol. 263, 375-386.

12. Krieglstein, J. (1969), Klin. Wochenschr. 47, 1125-1131.

13. Klotz, U., Antonin, K.-H. \& Bieck, P. R. (1976), J. Pharmacol. Exp. Ther. 199, 67-73.

14. Belpaire, F. M., Bogaert, M. G. \& Mussche, M. M. (1977), Europ. J. Clin. Pharmacol. 11, 27-32.

15. Garten, S. \& Wosilait, W. D. (1971), Biochem. Pharmacol. 20, 1661-1668.

16. Ehrnebo, M., Agurell, S., Jalling, B. \& Boreus, L. O. (1971), Europ. J. Clin. Pharmacol. 3, 189-193. 
17. Yacobi, A. \& Levy, G. (1975), J. Pharm. Sci. 64, 16601664.

18. Scholtan, W. \& Schmid, J. (1962), Arzneim.-Forsch. 12, 741-750.

19. Ehrnebo, M. \& Odar-Cederlöf, I. (1975), Europ. J. Clin. Pharmacol. 8, 445-453.

20. O'Reilly, R. O. \& Kowitz, P. (1967), J. Clin. Invest. 46, 829-837.

21. Niedner, R., v. Oettingen, U. \& Meyer, F. (1973), Int. J. Clin. Pharmacol. 82, 160-166.

22. Kurz, H. (1964), Naunyn-Schmiedebergs Arch. Pharmakol. $247,164-179$.

23. Singhvi, S. M., Heald, A. F., Gadebusch, H. H., Resnick, M. E., Difazio, L. T. \& Leitz, M. A. (1977), J. Lab. Clin. Med. 89, 414-419.

24. Andreasen, F. \& Jakobsen, P. (1974), Acta Pharmacol. Toxicol. 35, 49-57.

25. Andreasen, F. (1973), Acta Pharmacol. Toxicol. 32, 417-429.
26. Bird, A. E. \& Marshall, A. C. (1967), Biochem. Pharmacol. 16, 2275-2290.

27. Foged, L. Husted, S. \& Andreasen, F. (1976), Acta Pharmacol. Toxicol. 39, 312-320.

28. Seiler, K. \& Duckert, F. (1968), Thrombos. Diath. Haemorrh. 19, 389-396.

29. Paschen, K., Bautz, W. \& Bohner, J. (1976), Arzneim.Forsch. 26, 2137-2141.

30. Hooper, W. D., Dubetz, D. K., Bochner, F., Cotter, L. M., Smith, G. A., Eadie, M. J. \& Tyrer, J. H. (1975), Clin. Pharmacol. Ther. 17, 433-440.

31. Krieglstein, I. \& Kuschinsky, G. (1968), Arzneim.-Forsch. $18,287-289$.

32. Scatchard, G. (1949), Ann. N. Y. Acad. Sci., 51, 660-672.

33. Scholtan, W. (1964), Arzneim.-Forsch. 14, 348-356.

34. Scholtan, W. (1962), Makromol. Chem. 54, 24-59.

35. Klotz, I. M. \& Ayers, I. (1953), Discuss. Faraday Soc. 13, 189-196.
Ass. Prof. Dr. A. Kinawi Ostpreußendamm 111 D-1000 Berlin 45 